\title{
Physical and Emotional Impacts of the Caregiving Experience
}

\author{
Pamela R. Cosper ${ }^{\mathrm{a}}$ and Cindy L. Moyer ${ }^{\mathrm{a}}$
}

\begin{abstract}
Informal caregiving has become a widespread phenomenon, with family members and friends providing care for patients who have chronic or terminal illnesses and prefer to remain in their homes. The physical and emotional impacts of this unpaid work upon the caregiver can range from minor to severe. This study sought to identify qualities about the caregiving situation that may relate to the presentation of physical and/or emotional symptoms in the caregiver through the use of a researcher-generated survey with a non-random population in rural Pennsylvania. The authors hypothesized that frequency, duration, and invasiveness of the caregiving situation would impact upon the caregiver. Results of the study corroborated those of national studies that linked negative physical and emotional effects with increased frequency and duration of care. However, no correlation was found between positive emotions and the variables tested.
\end{abstract}

Keywords: Caregiving, Caregiver, Family caregiver, At-home care, Informal caregiving

\section{Introduction}

According to the National Alliance of Caregivers (NAC, 2009), "caregivers are defined as those who provide unpaid care to an adult or a child with special needs..." (p. 2). While this is a noble undertaking, quite often, informal caregiving is provided at the physical, emotional, and financial expense of the caregiver (Brewer \& Chu, 2008; NAC, 2009; PerrigChiello \& Hutchison, 2010). Like non-caregiving individuals, family caregivers are subjected to everyday stressors. This process frequently unfolds while the caregiver is also fulfilling the role of child, spouse, parent, etc., thus the aptly named Sandwich Generation (Jordan \& Cory, 2010; Ugwu, 2010). In addition to the demands of attending to a loved one, at-home caregivers may also perform or assist in the activities of daily living for the patient, such as bathing, dressing, feeding, incontinence care, etc. The cumulative effect on the caregiver's physical and emotional wellbeing often results in exhaustion, stress-related illness, and mood changes, among others.

The purpose of this study was to understand how the demands of the caregiving role affected caregivers physically and emotionally.

\section{Review of Related Literature}

Healthcare services (hospital, nursing home, hospice, etc.) provided to individuals with age-related illness or chronic health conditions consume a great deal of resources from Medicare and Medicaid (Institute of Medicine [IOM], 2008). According to the National Alliance for Caregiving, in collaboration with AARP (2009, p. 4): "In the [previous] 12 months, an estimated 65.7 million people in the U.S. [had] served as unpaid family caregivers to an adult or a child", with a staggering $31.2 \%$ of households having an individual who had provided unpaid caregiving within the same time period (p. 4). Houser and Gibson, on behalf of the AARP Public Policy Institute (2008), state: "The estimated economic value of their unpaid contributions was approximately $\$ 375$ billion in 2007, up from an estimated $\$ 350$ billion in 2006" (p. 1).
Although home health and hospice agencies provide care in private homes, they do not provide around-the-clock care. Supplemental assistance is often needed; however, this can present a financial burden for the patient or the family. The patient receiving care frequently prefers to reside in his/her home. Thus, the patient's spouse, child, sibling, friend, or other individual undertakes the role of informal, unpaid caregiver, thereby assuming not only the financial burdens of care, but the physical and emotional effects as well.

While numerous studies recognize that informal caregiving provides a tremendous financial adjunct in the care of veterans, children with special needs, individuals with intellectual and developmental disabilities, and aging Americans (Brewer \& Chu, 2008; Evercare \& NAC, 2007, 2009; Houser \& Gibson, 2008; Vitaliano \& Katon, 2006), to date, no legislation exists to support these unpaid caregivers. Though the economic impact is staggering, it was beyond the scope of this study. However, given the overwhelming numbers of individuals who provide at-home care in America, the authors chose to focus on the physical and emotional impacts of caregiving upon the caregiver, specifically upon those whose loved ones received services from local home health or hospice agencies in rural Pennsylvania.

Many studies have reported on the negative effects of caregiving, such as: declining health of the caregiver, stress, depression, increased financial burden, and impaired social and vocational relationships (Brewer \& Chu, 2008; Evercare \& NAC, 2007, 2009; Houser \& Gibson, 2008; NAC \& AARP, 2009; Perrig-Chiello \& Hutchison, 2010; Vitaliano \& Katon, 2006). In order to augment these findings, the researchers in the present study examined the relationships between the frequency, duration and invasiveness of care and the wellbeing of the caregiver. Hunt (2003) found that more recent research has been focused on the positive impacts of caregiving suggesting "supporting family caregivers ...[may]... improve quality of care and quality of life for both patients and family caregivers" (p. 31). In their caregiver assessment, Monin and Schulz (2010) theorized that the stage of caregiving (early, middle, or late in the disease process) 
could be an important indicator of positive impacts in the caregiving experience, with more positive effects reported by those providing care in the early stages of illness, possibly because the caregiver may feel s/he has more control in helping the care recipient at this early phase. Additionally, Ekwall and Hallberg (2007) found that a higher number of weekly caregiver hours actually indicated a higher level of caregiver satisfaction.

Likewise, this study sought to ascertain the relationship between caregiving and the positive or negative impacts experienced by the caregiver.

To summarize, the following research questions were considered:

- Does the duration of family caregiving impact the physical or emotional wellbeing of the caregiver?

- Does the frequency of physical or household tasks performed by the caregiver impact the physical or emotional wellbeing of the caregiver?

- Does the provision of high invasive/high intimacy types of duties (e.g., ostomy care, wound care, incontinence care, etc.) have an impact upon the physical or emotional wellbeing of caregivers?

- Do low invasive/low intimacy types of duties (e.g., feeding, medication administration, grooming) have an impact upon the physical or emotional wellbeing of caregivers?

In examining the above questions, the researchers developed several hypotheses that were evaluated in the study. They were:

- $\quad$ Providing informal caregiving for extended periods of time (less than 6 months to more than one year) will have an impact on the physical states of the caregiver.

- $\quad$ Providing informal caregiving for extended periods of time (less than 6 months to more than one year) will have an impact on the emotional states of the caregiver.

- Providing informal caregiving more frequently (more than 3 days each week) will have an impact on the physical states of the caregiver.

- Providing informal caregiving more frequently (more than 3 days each week) will have an impact on the emotional states of the caregiver.

- Informal caregivers who engage in high invasive/high intimacy types of duties (e.g., ostomy care, wound care, incontinence care, etc.) will experience physical impacts.

- Informal caregivers who engage in high invasive/high intimacy types of duties (e.g., ostomy care, wound care, incontinence care, etc.) will experience emotional impacts.

- Informal caregivers who engage in low invasive/low intimacy types of duties (e.g., feeding, grooming, medication administration, etc.) will experience satisfaction.

The independent variables in the above hypotheses were, respectively: the duration of caregiving; the frequency of caregiving; and for the last three, the invasiveness and intimacy of the caregiving duty, to a greater or lesser extent.
These contributing factors in the caregiving experience were expected to be quite diverse given the myriad caregiving situations in this study and so were considered independent based on that diversity. The dependent variables are the physical and emotional impacts of caregiving for the first six hypotheses, with satisfaction being the dependent variable for the last hypothesis. Dependent variables in this study were the measurable outcomes of the influence of the independent variables upon family caregivers.

Some examples of physical impacts were increased backaches, headaches and prescription drug use. Some of the possible emotional impacts experienced by the caregiver included increased anger, guilt, fear, and depression. Satisfaction was defined as the caregiver experiencing increased levels of compassion, contentment, dependability, fortune, fulfillment, gratitude, helpfulness, hope, love, peace, productivity, and supportiveness. Concurrently, in experiencing satisfaction, the caregiver would have decreased feelings of anger, anxiety, depression, exhaustion, fear, frustration, guilt, helplessness, isolation, worry and feeling overwhelmed and unappreciated. Extraneous variables (factors which the researchers were not measuring or evaluating) that may have affected the outcomes predicted were: the caregiver's own health, age, ethnicity, relationship to care recipient, and care recipient's health condition.

\section{Methodology}

\section{Sample}

Informal caregivers whose loved ones were patients of rural Pennsylvania home health and hospice agencies were recruited for this study. These facilities were approached with a recruitment letter explaining the purpose of the study and the researchers' desire to engage in an anonymous, confidential sampling method. Once agencies consented to participation, in lieu of an informed consent document, potential respondents received a Participant Letter, which explained the purpose of the survey, potential risks of participation, possible benefits of the research, and the right to refuse participation. This explanatory letter was used instead of a Participant Permission Letter in order to maintain the strictest standards of anonymity in the sampling process. All participants were treated in accordance with Federal Regulations (45 CFR 46) (Office of Human Subjects Research's Protection of Human Subjects Regulation, 2005), the Institutional Review Board (IRB) of Marywood University (Marywood University, 2011), and the National Association of Social Workers (NASW) Code of Ethics (1996, revised 2008).

Because a non-probabilistic convenience sample (participants not chosen randomly but from a select group of patient censuses) was used for this study, the researchers did not have a sampling plan beyond asking agencies to mail the survey packets to their current patient census and deceased patients' families. The authors solicited the assistance of rural Pennsylvania home health and hospice agencies and hospitals. The recruitment strategy succeeded in establishing collaboration with two home health and hospice agencies. The total number of survey packets mailed to prospective participants was 645. One hundred forty-four individuals returned surveys. However, of this 144, 11 were discarded due to failure to complete the survey, excessive missing data, 
survey completion by the care recipient instead of the caregiver, or survey completion by a sole individual caring for $\mathrm{him} / \mathrm{herself}$. This resulted in a $21 \%$ response rate, which was less than ideal.

\section{Definitions}

For purposes of this study, the researchers defined caregiving as the act of providing physical or supportive care to an individual who was receiving home care or hospice services at the time of data collection, January 1, 2012 February 15, 2012. Additionally, the population who had received hospice services between July - December, 2011 was included to increase sample size. Caregiving included household or supportive duties such as, but not limited to, laundry, meal preparation, grocery shopping, yard maintenance, financial management, or transportation to medical appointments. The caregiver was defined as the person who provided physical care and/or performed household duties for the care recipient. The care recipient was defined as the person who was admitted to home health or hospice services and had received assistance from the caregiver.

\section{Instrumentation and procedures}

A researcher-generated survey (Appendix A) was created consisting of 18 questions, including demographics of the caregiver, care recipient, duration of care, types of care provided and the physical and emotional impacts upon the caregiver. Likert scales were utilized to assess the types and frequencies of various services provided, as well as the types and frequencies of the physical and emotional symptoms experienced by the caregivers. The instrument would produce similar results if respondents completed the survey multiple times, thereby giving it internal consistency reliability.

Based on the ecological perspective, which involves the reciprocal relationship between person and environment, the researchers expected that the type, duration, and frequency of caregiving tasks would support a concurrent validity in the measurements of type and frequency of physical and emotional impacts. The survey was also designed to collect extraneous variables that may have impacted the caregiver experience. Because the survey instrument was not thoroughly vetted, three Masters-level professionals reviewed it for appropriateness of use. Data was collected from January 1 - February 15, 2012. Participants were provided with self-addressed, stamped envelopes to facilitate the return of their completed surveys to the researchers. Participants were asked to assess changes in their physical status through the duration of their caregiving experience using a nominal level of measurement (this is not a concern for me, no change during caregiving, got worse during caregiving, or got better during caregiving). Participants were also asked to assess their emotional response(s) to caregiving duties by reporting the frequency of occurrence of both positive and negative emotions. Specifically, participants rated their emotional responses based on a Likert scale using an ordinal level of measurement (never, rarely, sometimes, often, all the time). The survey took approximately 15 minutes to complete.

Upon completion of the study, all surveys were placed in a locked, metal file cabinet in the home of researcher Moyer, the only person with access. All records will be destroyed on June 1, 2014 by shredding of paper documents and deletion of electronic records.

Participants were not debriefed following the completion and return of surveys due to the anonymous nature of the data collection process. However, they were offered the opportunity to request a copy of the final study from their respective home health and hospice agencies. No incentives were provided to participants other than the opportunity to participate in a survey about caregiver stressors that could lead to agency and/or community support programs and services for caregivers.

\section{Data Analysis}

Data collected were analyzed using the Statistical Package for the Social Sciences (SPSS) software program. Demographic analysis of the data showed that $79 \%$ of caregivers in this study were female; $21 \%$ were male. Combining male and female caregivers yielded the modal score (most frequently occurring score) of $41 \%$ in the 51-65 year age range. In terms of those receiving care, 53\% were females; $47 \%$ were male. The caregivers and recipients' distributions of gender and age ranges are shown in Figure 1

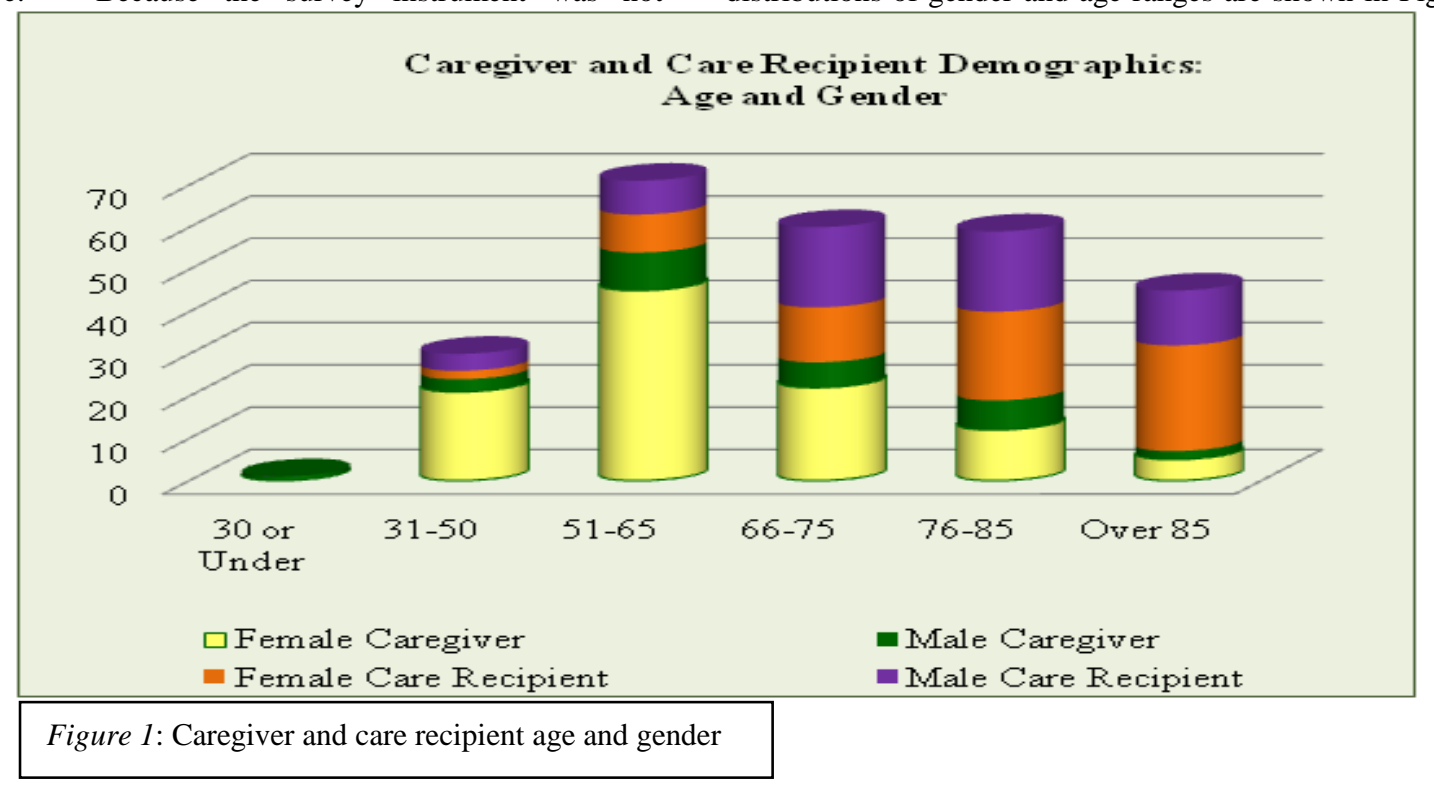


Evaluating the physical impacts of the caregiving experience against the duration of caregiving showed no significant relationship between the variables, indicating that duration was not a factor in physical impacts of caregiving in this study. However, in testing the emotional impacts against duration, three variables were found to have significant relationships: contentment $\left(x^{2}=22.951, d f=8, p<.003\right)$, fear $\left(x^{2}=16.463, d f=8, p<.036\right)$, and guilty $\left(x^{2}=18.909, d f\right.$ $=8, p<.015)$. These results implied that caregivers who provided care for longer periods of time experienced not only higher rates of contentment but also higher rates of fear and guilt.

Measuring frequency against impacts on the physical wellbeing of the caregiver showed interesting results. Due to the design of the question measuring the physical impacts, it was not possible to collapse the negative and positive effects into separate composite variables. Respondents were asked whether a physical symptom improved or worsened during caregiving. Survey participants could also choose "no change" or "not a concern for me" with each physical symptom presented thereby rendering responses without assigning a negative or positive value. Therefore, the frequency variable was run against each of the physical impacts individually.

In terms of the negative physical impacts upon the caregiver (e.g., backaches, headaches, fatigue, inadequate sleep, etc.), statistically significant results were found at the $p$ $<.05$ level for 46 variables between the aforementioned types of physical symptoms and the types and frequencies of caregiving provided (e.g., laundry, dressing, feeding, ostomy care, etc. provided: never, 1-3 days, 4-6 days, or every day). The combination of type and frequency of care with certain physical symptoms showed overwhelming overlap with several physical impacts on the caregiver. Of these 46 significant results, Table 1 shows a representative sampling, as the inclusion of the entire data set was space prohibitive.

Table 1

Significant cross tabulation results of caregiving duties and physical symptoms experienced

Caregiving performed

Physical impact

$x^{2}$

$d f \quad p$ value

Physical Care:

\begin{tabular}{|c|c|c|c|c|}
\hline Application of skin preparations & Feeling tired & 23.718 & 9 & 0.005 \\
\hline Bathing/showering & Endurance/strength & 21.205 & 9 & 0.012 \\
\hline Feeding & Feeling tired & 21.860 & 9 & 0.009 \\
\hline Giving nasal sprays & $\begin{array}{l}\text { Backache } \\
\text { Not sleeping enough } \\
\text { Trouble falling/staying asleep }\end{array}$ & $\begin{array}{l}21.596 \\
20.647 \\
22.482\end{array}$ & $\begin{array}{l}9 \\
9 \\
9\end{array}$ & $\begin{array}{l}0.010 \\
0.014 \\
0.007\end{array}$ \\
\hline Giving breathing treatments & Weight loss & 40.320 & 9 & 0.000 \\
\hline Incontinence care & Physical strength & 21.130 & 9 & 0.012 \\
\hline Ostomy care & Sleeping too much & 36.331 & 9 & 0.000 \\
\hline \multirow[t]{2}{*}{ Transfers } & Feeling tired & 21.636 & 9 & 0.010 \\
\hline & Physical strength & 20.590 & 9 & 0.015 \\
\hline
\end{tabular}

\section{Household/Supportive Care:}

\begin{tabular}{|c|c|c|c|c|}
\hline Meal preparation & Use of prescription medications & 27.551 & 9 & 0.001 \\
\hline \multirow[t]{3}{*}{ Shopping/errands } & Feeling tired & 27.761 & 9 & 0.010 \\
\hline & Not sleeping enough & 33.382 & 9 & 0.000 \\
\hline & Sleeping too much & 25.356 & 9 & 0.003 \\
\hline \multirow[t]{3}{*}{ Laundry } & Backache & 27.622 & 9 & 0.001 \\
\hline & Eating/nutrition & 23.475 & 9 & 0.005 \\
\hline & Feeling tired & 24.232 & 9 & 0.004 \\
\hline \multirow[t]{2}{*}{ Housecleaning } & Medical condition & 22.670 & 9 & 0.007 \\
\hline & Use of prescription medications & 20.600 & 9 & 0.015 \\
\hline \multirow[t]{2}{*}{ Banking/bill paying } & Ability to concentrate & 40.892 & 9 & 0.000 \\
\hline & Physical strength & 28.194 & 9 & 0.005 \\
\hline
\end{tabular}

Note: All results were evaluated at the $\mathrm{p}<.05$ level.

${ }^{\mathrm{a}} \mathrm{n}=133$.

ISSN: 2167-1907

www.jofsr.com 
breathing treatments, incontinence care, mobility, meal preparation, and yard work/maintenance (Figure 2). These results implied that the more frequently these types of care were provided, the higher the total negative emotion score. Only one positive emotion variable labeled, "participating in social engagements", showed statistical significance when run against frequency $\left(x^{2}=125.413, d f=90, p<.008\right)$. The implication being that the more often caregivers participated in social engagements with the care recipient, the more positive emotion they experienced. significant relationships, all at the $p<.05$ level: medications administered by injection, giving nasal sprays, giving statistically significant results, albeit to a lesser extent. Unlike the physical impact scale, however, the emotion scale lent itself to collapsing the negative emotions and positive emotions into separate composite variables (total negative emotion variable and total positive emotion variable).

The frequency of caregiving tasks evaluated against the negative emotion composite variable yielded several

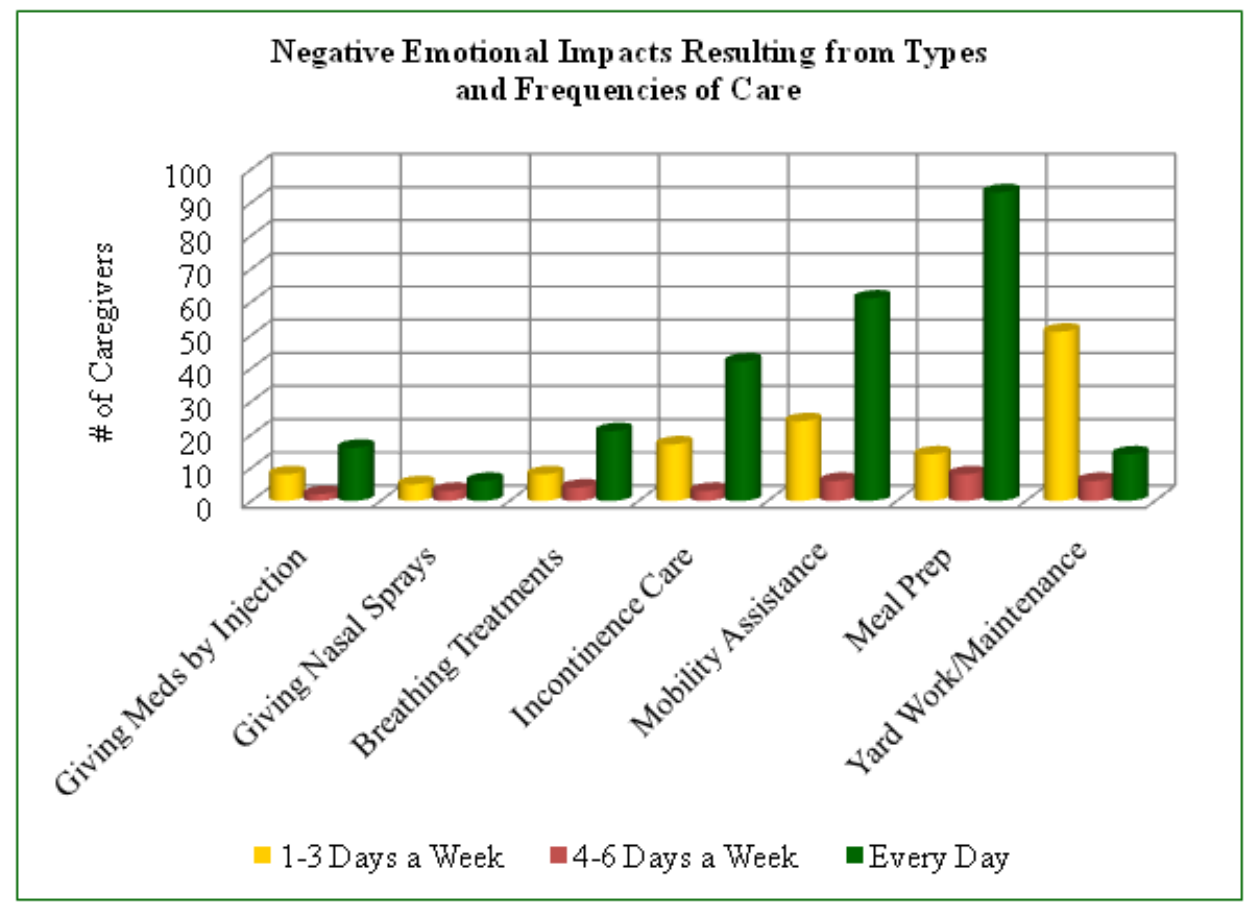

Figure 2: Significant correlations between type and frequency of care provided tested against the negative emotion composite variable.

\section{Discussion}

\section{Impressions}

The statistical data on caregiver demographics corresponds with the figures reported at the national level (NAC \& AARP, 2009), although the most frequently reported caregiver age range for this study, 51-65 years, was slightly greater than the national average of 48 years. This discrepancy may be accounted for by the limited sample size of the current study compared to the national survey.

Analyses of the care recipient demographics also differed somewhat from data gathered in the national survey (NAC \& AARP, 2009). Nationwide, 62\% of care recipients are female, while this survey found that figure to be $53 \%$. Likewise, the current study had divergent results in terms of care recipient age, with the most often-reported age bracket between 76-85 years. Nationally, the average age is 61 years. In terms of the relationship between the caregiver and care recipient, the highest frequency reported was husband (34\%), followed by mother $(25 \%)$ and wife $(14 \%)$. The overall percentage of individuals caring for a relative was $97 \%$, which was somewhat above the national rate of $86 \%$. Likewise, a discrepancy was found between caregivers in this study providing care for 12 months or more (54\%) and the national survey, which was $65 \%$.

What follows below is a summary of the findings relevant to the hypotheses posited by the researchers:

- The most often reported physical ailments experienced by caregivers were: arthritis/bone fractures (45\%), followed distantly by allergies $(24 \%)$, other disorders (e.g., Crohn's Disease) (20\%), diabetes (18\%), depression (17\%) and heart failure/heart disease (17\%). This corroborates the data reported by several studies (Brewer \& Chu, 2008; Evercare \& NAC, 2007, 2009; Houser \& Gibson, 2008; NAC \& AARP, 2009; PerrigChiello \& Hutchison, 2010; Vitaliano \& Katon, 2006).

- The results of the current study found no relationships between the positive emotion variables and duration of care at the less than 6 months or the 6 month to 12 month time periods. Only one correlation was found for contentment/happiness at the duration variable of 12 months or more. 
- $\quad$ The researchers found no significant relationships in this study between the duration of caregiving and the physical impacts experienced by the caregiver, thereby leaving the authors unable to reject the null hypothesis for this research question.

- The duration of caregiving produced three significant relationships when evaluated against the emotional impacts of caregiving. These results supported the hypothesis that providing informal caregiving for extended periods of time would have an impact on the emotional wellbeing of the caregiver, albeit to a lesser extent than anticipated by the researchers.

- Additionally, only one variable measuring positive emotions was found to be relevant: contentment $(p<$ .003). However, two variables assessing negative emotional impacts yielded statistical significance: fear ( $\mathrm{p}$ $<.036)$ and guilt $(p<.015)$.

- In terms of the frequency of the caregiving experience impacting upon the physical and emotional wellbeing of respondents, results were in alignment with what one could expect from a physically demanding set of tasks. For instance, backaches were more often reported by those individuals who provided assistance to care recipients in transferring (e.g., from bed to chair or wheelchair to toilet).

- Similarly, the frequency variable showed significance when related to the negative emotion composite variable. The results of these analyses support the hypothesis posited by the authors that caregivers who provide high invasive/high intimate intimacy types of care (e.g. bathing/showering, incontinence and ostomy care) would experience emotional impacts.

- The same may be said of the positive emotion composite variable of participating in social engagements, which, when provided more frequently to the care recipient, would have an uplifting impact upon the caregiver. This result lends support to the researchers' hypothesis that those engaging in low invasive/low intimacy types of caregiving would experience satisfaction.

\section{Limitations of study}

The researchers assumed that caregivers might report both negative and positive impacts on their physical and/or emotional wellbeing, with variation in individual experiences. Some caregivers may have failed to respond due to the overwhelming nature of the caregiving they were providing during the period of data collection. This potential exclusion of individuals may have limited response rates from caregivers with higher frequency, longer duration, or higher levels of intimate/invasive of caregiving. While it is feasible that the majority of participants experienced no physical and minimal emotional impacts from the caregiving experience, it may be more likely that the relatively low response rate influenced the results or the survey design itself was lacking in the capacity to measure such relationships. Other limitations of the study included the lack of a large-print version for low-vision caregivers and the physical health of the caregiver (e.g., severe arthritis may have been an impediment to completing a written survey).

The length of the survey instrument may have deterred some caregivers from participating. While it took approximately 15 minutes to complete, the number of pages (nine) may have dissuaded some prospective respondents.
Still other potential participants may have been overwhelmed with the performance of caregiving duties, professional and personal obligations, which left inadequate time or interest in responding to the survey.

Health Insurance Portability and Accountability Act (HIPAA) concerns and time limitations of Institutional Review Boards (IRB) raised by some of the solicited agencies also limited this study. In other cases, workload of agency employees available to address and mail envelopes may have prevented collaboration. Although the survey packets were collated and placed in an outer envelope with sufficient postage, the collaborating agencies and hospitals needed to address the envelopes for mailing. The researchers recognize that this limitation could not be remedied without compromising the anonymous nature of the study design and requesting patient census mailing lists to mail packets on their own.

The homogenous population of rural Pennsylvania may have further limited this study. Also, because the researchers relied upon a non-probabilistic convenience sample rather than a random sample, the generalizability of any findings was limited. A correction of this limitation would be to randomly survey family caregivers in urban portions of Pennsylvania and other states.

Further limiting this study was the low response rate and in many cases, the volume of missing data in several of the questions. Both of these factors decreased the confidence level of statistical analyses.

\section{Implications}

Through the course of this research, the authors learned the value of a thoroughly vetted survey instrument. While the questionnaire developed by the researchers was critically reviewed by approximately 15 individuals and went through numerous iterations, it nonetheless presented participants with a few challenges in terms of responding to all of the questions. Refinement of the instrument would, ideally, reduce the amount of missing data and increase confidence levels of the data and the inferential statistics.

It also became evident that future instructions to participating agencies should be clarified to read informal, unpaid caregivers are being recruited. In one instance, survey packets were inadvertently sent to private-duty caregivers engaged in professional caregiving. None of these surveys were used in the tabulation of results. Also, information should be added to the Participant Letter explicating two issues: a) if the residence to which the mailing was sent does not include a caregiver, please disregard the mailing and discard the survey; $b$ ) if the mailing was inadvertently sent to a care recipient, it should be passed along to the individual's caregiver.

The research could also be refined by narrowing the population of caregivers to those who had cared for hospice patients during a specified period of time. This would serve two purposes: providing a clearer pool of respondents and cost containment of the project.

The researchers also learned the value of planning adequate time to allow for the IRB processes of individual agencies being solicited for participation in the study.

The overall results of this study lend support to the data published by the NAC and AARP (2009). Although the sample size of this project was small, the conclusions that may be drawn are nonetheless compelling. 
Directions for future research may include further inquiry into the burden of caregiving specifically in terms of high invasive/high intimacy versus low invasive/low intimacy types of caregiving. Additionally, it would be intriguing to examine in depth the disparity of negative emotions reported between female and male caregivers. Although data was collected on acute and chronic disease comorbidities for both the caregiver and the care recipient, the researchers found it necessary to limit the scope of the project. Thus, it would be interesting to explore this aspect of the caregiving experience further.

This type of research can be used to drive legislative action that would better fund care of the elderly and those with disabilities. More importantly, it provides concrete evidence to support legislation that would fund the millions of informal caregivers across America.

\section{References}

Brewer, L., \& Chu, D. (2008). Caregivers at risk: The implications of health disparities. Journal of Loss and Trauma, 13, 205-221. doi: $10.1080 / 15325020701769246$

Ekwall, A. K., \& Hallberg, I. R. (2007). The association between caregiving satisfaction, difficulties and coping among older family caregivers. Journal of Clinical Nursing, 16, 832-844. doi: 10.1111/j.13652702.2006.01382.x

Evercare by UnitedHealthcare, \& the National Alliance on Caregiving (NAC). (2009, April 28). The Evercare ${ }^{\circledR}$ survey of the economic downturn and its impact on family caregiving. Minnetonka, MN: Author.

Evercare (in collaboration with the National Alliance for Caregiving [NAC]). (2007, November). Evercare ${ }^{\circledR}$ study of family caregivers - what they spend, what they sacrifice; The personal financial toll of caring for a loved one. Minnetonka, MN: Author.

Houser, A., \& Gibson, M. J. (2008). Valuing the invaluable: The economic value of family caregiving (2008
Update). Washington, DC: AARP Public Policy Institute.

Hunt, C. K. (2003). Concepts in caregiver research. Journal of Nursing Scholarship, 35(1), 27-32.

Institute of Medicine. (2008). Retooling for an aging America: Building the health care workforce (Free Executive Summary). Washington, DC: The National Academies Press.

Jordan, C., \& Cory, D. (2010). Boomers, boomerangs, and bedpans. National Social Science Journal, 34(1), p. 79-84.

Marywood University, Institutional Review Board. (2011). Protecting human research participants. Retrieved from http://www.marywood.edu/irb/

Monin, J. K., \& Schultz, R. (2010). The effects of suffering in chronically ill older adults on the health and wellbeing of family members involved in their care: The role of emotion-related processes, GeroPsych, 23(4), 207-213. doi: 10.1024/1662-9647/a000024

National Alliance for Caregiving (in collaboration with AARP). (2009). Caregiving in the U.S. (Executive Summary). Bethesda, MD: Author.

National Association of Social Workers. (1996, revised 2008). NASW code of ethics. Retrieved from http://www.socialworkers.org/pubs/code/code.asp

National Institutes of Health, Office of Human Subjects Research's Protection of Human Subjects Regulation, 45 C.F.R. 46 (2005). Retrieved from http://ohsr.od.nih.gov/guidelines/45cfr46.html\#top

Perrig-Chiello, P., \& Hutchison, S. (2010). Family caregivers of elderly persons; A differential perspective on stressors, resources, and well-being. GeroPsych, 23(4), 195-206. doi: 10.1024/1662-9647/a000025

Ugwu, L. (2010). Employed "Sandwich" Generation women: Coping with work/family conflicts. European Journal of Social Sciences, 15(3), p. 396-410.

Vitaliano, P. P., \& Katon, W. J. (2006). Effects of stress family caregivers: Recognition and management. Psychiatric Times, 13(7), 24-28. 